\title{
THE NONEXISTENCE OF UNIVERSAL INVARIANT MEASURES
}

\author{
C. RYLL-NARDZEWSKI AND R. TELGÁRSKY
}

\begin{abstract}
It is shown that for arbitrary nontrivial $\sigma$-finite measure defined on all subsets of a group there are at most countably many left translations of that measure that are mutually equivalent in the sense of the absolute continuity.
\end{abstract}

This note was inspired by the theorem of Erdös and Mauldin [3] on the nonexistence of invariant universal measures on uncountable groups. Slightly modifying the reasonings of [3] we get a fairly general lemma on invariance properties of $\sigma$-ideals of subsets of an arbitrary group. As a corollary we obtain a refinement of the theorem from [3], as follows: for arbitrary nontrivial $\sigma$-finite measure defined on all subsets of a group there are at most countably many left translations of that measure that are mutually equivalent in the sense of the absolute continuity. Besides we mention results of a similar kind for semigroups and for finitely additive measures.

One says that a $\sigma$-ideal $I$ of subsets of a set $G$ satisfies the countable chain condition (CCC) if every uncountable family of pairwise disjoint subsets of $G$ contains an element of $I$.

The next assertion is essentially due to $\mathrm{S}$. Ulam [5]:

Proposition. If $I$ is a proper $\sigma$-ideal of subsets of a set $G$ satisfying $C C C$, then $G$ is not the union of $\aleph_{1}$ sets from $I$.

The proof is based on an application of the famous Ulam matrix of type $\kappa_{0} \times \kappa_{1}$.

Now let us assume that the set $G$ is a group. We shall say that an element $g$ of $G$ is left-side admissible with respect to $I$ (in short: admissible) if for each $E \subset G$ we have $E \in I$ iff $g E \in I$. It is easy to see that the set $A$ consisting of all admissible elements of $G$ is a subgroup of $G$.

Lemma. Under the above assumptions (Proposition) $A$ is countable.

Proof. Suppose, a contrario, that $A$ is not countable. Then $A$ contains a subgroup $H$ of the cardinality $\aleph_{1}$. Let $S$ be a selector from the family $\{\mathrm{Hg}$ : $g \in G\}$ of right-side cosets of $H$. We have $h_{1} S \cap h_{2} S=0$ for $h_{1} \neq h_{2}, h_{1}$, $h_{2} \in H$. Hence $\{h S: h \in H\}$ is an uncountable family of pairwise disjoint

Received by the editors July 5, 1977.

AMS (MOS) subject classifications (1970). Primary 28A70; Secondary 04A10.

Key words and phrases. Left-invariant measure, measurable cardinal. 
subsets of $G$. Since the ideal $I$ satisfies CCC, it follows that $h_{0} S \in I$ for some $h_{0} \in H \subset A$, and consequently $h S \in I$ for each $h \in H$. However, $G=H S$ $=\bigcup\{h S: h \in H\}$ and this is a contradiction to the Proposition.

We say that measures $\mu_{1}$ and $\mu_{2}$ are equivalent, in symbols: $\mu_{1} \sim \mu_{2}$, if their ideals of nullsets are identical. If $\mu$ is a measure on a group $G$ and $g \in G$, then ${ }_{g} \mu$ denotes the measure defined by setting ${ }_{g} \mu(E)=\mu(g E)$ for each $E \subset G$.

By the Lemma we immediately get

THEOREM. If $\mu$ is a nontrivial $\sigma$-finite measure defined on all subsets of a group $G$, then the set $A=\left\{g \in G:{ }_{g} \mu \sim \mu\right\}$ is countable.

That theorem is a refinement of the above-mentioned result of Erdös and Mauldin [3]: Suppose $G$ is an uncountable group and $\mu$ is a $\sigma$-finite countably additive left-invariant measure defined on all subsets of $G$. Then $\mu$ is trivial.

Problem. Let $G$ be an uncountable abelian semigroup with cancellation law. Does there exist a nontrivial $\sigma$-finite measure $\mu$ defined on all subsets of $G$ such that $\mu(g+E)=\mu(E)$ for each $g \in G$ and $E \subset G$ ?

Let us note that in some cases the answer is no, e.g., for the additive semigroup of nonnegative reals.

At the end of this note let us mention a few remarks on the analogous results for finitely additive measures.

1. Let $G$ be an abelian semigroup. Then there exists a finitely additive measure $\mu$ defined on all subsets of $G$ such that $\mu(G)=1$ and $\mu(\{x \in G$ : $g+x \in E\})=\mu(E)$ for each $g \in G$ and $E \subset G$.

That assertion is a consequence of the Kakutani-Alaoglu Fixed Point Theorem (cf. [2]).

2. In view of the paradoxical decomposition of Hausdorff-Banach-Tarski (cf. [4]), for nonabelian groups left-invariant finitely additive universal measures need not exist in general.

3. Let $G$ be a group and let $g_{0}$ be a fixed element of $G$ different from the neutral element. Then there is no nontrivial $g_{0}$-left-invariant finitely additive measure defined on all subsets of $G$ and taking values 0 or 1 only.

The last assertion is an immediate consequence of the following theorem of Katětov (cf. [1]): If $f$ is a map from a set $X$ into itself without fixed points, then there exists a decomposition of $X$ onto three sets $X_{1}, X_{2}$ and $X_{3}$ such that $X_{i} \cap f\left(X_{i}\right)=0$ for $i=1,2$, 3. (We owe that observation to E. Grzegorek.) However, in the case of the left shift $g \mapsto g_{0} g$, the proof is much easier and it reduces to cyclic groups only.

\section{REFERENCES}

1. W. W. Comfort and S. Negrepontis, The theory of ultrafilters, Grundlehren math. Wiss., Bd. 211, Springer-Verlag, Berlin, 1974. 
2. N. Dunford and J. Schwartz, Linear operators, Vol. I, Interscience, New York, 1958.

3. P. Erdös and R. D. Mauldin, The nonexistence of certain invariant measures, Proc. Amer. Math. Soc. 59 (1976), 321-322.

4. F. P. Greenleaf, Invariant means on topological groups and their applications, Van Nostrand Reinhold, New York, 1969.

5. S. M. Ulam, Zur Masstheorie in der allgemeinen Mengenlehre, Fund. Math. 16 (1930), 141-150.

Institute of Mathematics, Technical University, 50-370 Wrockaw, Poland 